\title{
P48 Effects of Remote Ischaemic Preconditioning on Haemodynamic and Arterial Stiffness Parameters in Patients Undergoing Lower Limb Angiographic Procedure
}

Karl Kuusik*, Teele Kepler, Mihkel Zilmer, Jaan Eha, Mare Vähi, Kaido Paapstel, Jaak Kals

University of Tartu, Tartu, Estonia

\begin{abstract}
Objectives: Remote ischaemic preconditioning (RIPC) is a phenomenon that promotes protection of tissues and organs against ischaemia-reperfusion injury. RIPC has been shown to reduce myocardial and renal injury but its effect on arterial stiffness in patients undergoing lower limb digital subtraction angiography (DSA) is unknown. The aim of this study was to evaluate the effect of RIPC on arterial stiffness in patients with peripheral arterial disease (PAD) undergoing lower limb DSA.

Methods: In RIPC intervention, the blood pressure cuff on the arm was inflated to $200 \mathrm{mmHg}$ or to $20 \mathrm{mmHg}$ above systolic pressure and in sham intervention, to $20 \mathrm{mmHg}$, for four 5 -minute cycles at 5-minute intervals between the cycles. Changes in heart rate corrected augmentation index (AIx@75), augmentation index (AIx), carotid-femoral pulse wave velocity (PWV) and haemodynamic parameters were measured before and 24 hours after DSA.

Results: 111 (RIPC 54, sham 57) patients with symptomatic lower limb PAD scheduled for DSA were randomised. 102 patients (RIPC 47, sham 55 ) were included in final analysis. RIPC significantly improved AIx $(-5.46 \%$ in RIPC and $-1.45 \%$ in sham group; $p=0.045)$, but not AIx@75 (-4.88\% in RIPC and $-1.38 \%$ in sham group; $p=0.071)$ or PWV $(-0.41 \mathrm{~m} / \mathrm{s}$ in RIPC and $-0.27 \mathrm{~m} / \mathrm{s}$ in sham group; $p=0.741)$. In the RIPC group a significant reduction in AIx $(p=0.002)$ and AIx@75 $(p=0.003)$ was noted after stenting when compared to the sham intervention.

Conclusion: RIPC improves haemodynamic profile and modulates arterial stiffness following DSA procedure and might be more pronounced in patients after stent placement.
\end{abstract}

(c) 2019 Association for Research into Arterial Structure and Physiology. Publishing services by Atlantis Press International B.V. This is an open access article distributed under the CC BY-NC 4.0 license (http://creativecommons.org/licenses/by-nc/4.0/). 